\title{
Radiation hardness of GeSi heterostructures with thin Ge layers
}

\author{
J.P. Leitão ${ }^{\text {a, } *}$, N.M. Santos ${ }^{\text {a }}$, N.A. Sobolev ${ }^{\text {a }}$, M.R. Correia ${ }^{a}$, N.P. Stepina ${ }^{\text {a,b }}$, M.C. Carmo ${ }^{\text {a }}$, \\ S. Magalhães ${ }^{\text {c }}$, E. Alves ${ }^{c}$, A.V. Novikov ${ }^{d}$, M.V. Shaleev ${ }^{d}$, D.N. Lobanov ${ }^{\text {d }}$, Z.F. Krasilnik ${ }^{d}$ \\ ${ }^{a}$ Departamento de Física and I3N, Universidade de Aveiro, 3810-193 Aveiro, Portugal \\ ${ }^{\mathrm{b}}$ Institute of Semiconductor Physics, 630090 Novosibirsk, Russia \\ c Instituto Tecnológico e Nuclear, E.N. 10, 2686-953 Sacavém, Portugal \\ ${ }^{\mathrm{d}}$ Institute for Physics of Microstructures, GSP-105, 603950 Nizhny Novgorod, Russia \\ Received 30 June 2007; received in revised form 25 July 2007; accepted 28 August 2007
}

\begin{abstract}
The influence of defects on the optical properties of a single Ge quantum well deposited on a Si substrate and on a diode structure containing a Si/Ge multilayer structure was investigated. In order to change the density of optically active defects, the as-grown samples were exposed to post-growth treatments: atomic hydrogen passivation and irradiation with $2.0 \mathrm{MeV}$ protons to fluences in the range $2 \times 10^{12}$ to $1 \times 10^{14} \mathrm{~cm}^{-2}$. The optical and structural properties were investigated by photoluminescence and X-ray diffraction and reflection measurements. An unexpectedly high radiation hardness was observed for the as-grown Ge quantum wells.
\end{abstract}

(C) 2007 Elsevier B.V. All rights reserved.

Keywords: Ge/Si quantum wells; Radiation hardness; Photoluminescence

\section{Introduction}

The growth of $\mathrm{Si} / \mathrm{Ge}$ heterostructures and the study of their optical and structural properties have been a very active area of research in the last 30 years due to its potential contribution for the development of new electronic and optoelectronic devices [1]. The presence of defects on these structures critically influences their properties so that a possible use of them in harsh environments requires the study of their response under extreme working conditions.

Recently, high radiation hardness (RH) has been found for low dimensional structures based on different systems such as: InGaAs/GaAs [2-4], AlAs/GaAs [5] and Si/Ge [6-8]. The RH is higher for superlattices [6] and for quantum dots [2,3,7] when compared to quantum wells (QWs). Furthermore, the combination of a superlattice with quantum dots resulted in an even more enhanced RH [5,8].

An elevated RH of the QWs is not expected a priori because (i) for the above bandgap excitation, the radiation defects in the surrounding matrix compete with the QWs for the capture of photoexcited charge carriers; (ii) the carriers captured by the

\footnotetext{
* Corresponding author. Tel.: +351 234370 286; fax: +351 234424965 . E-mail address: joaquim.leitao@ua.pt (J.P. Leitão).
}

QWs are delocalized in two dimensions and can be captured by radiation defects created inside the QWs. Nevertheless, we have observed in this work an unexpectedly high $\mathrm{RH}$ of $\mathrm{Ge}$ QWs. Two sample types were studied, one with a single Ge QW deposited on a Si substrate and another one being a diode structure containing a Si/Ge multilayer grown on Si. Post-growth treatments (atomic hydrogen passivation and proton irradiation) were used in order to change the defect density. The optical and structural properties were investigated by photoluminescence and X-ray diffraction and reflection measurements.

\section{Experimental}

The diode structure (sample A) was grown by molecular beam epitaxy (MBE) at $600^{\circ} \mathrm{C}$ on a $\mathrm{Si}\left(\begin{array}{lll}0 & 1)\end{array}\right)$ and consisted of 20 periods. A Ge and a Si layer with nominal thicknesses of 3.5 monolayers (ML) and $20 \mathrm{~nm}$, respectively, were deposited in each period. Below the multilayer structure and on top of the substrate, $200 \mathrm{~nm}$ of Si heavily doped with B and $50 \mathrm{~nm}$ of undoped $\mathrm{Si}$ were deposited. The sample was terminated with $50 \mathrm{~nm}$ of undoped $\mathrm{Si}$ and $200 \mathrm{~nm}$ of Si heavily doped with Sb. In the other sample (sample B), a Ge layer with a nominal thickness

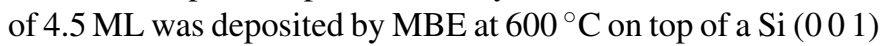
substrate covered by a $100 \mathrm{~nm}$ thick Si buffer layer. The sample was terminated by a $80 \mathrm{~nm}$ thick Si capping layer. Two post- 
growth treatments were used: passivation with atomic hydrogen, performed in a CVD reactor at $T=100{ }^{\circ} \mathrm{C}$ for $30 \mathrm{~min}$, and the irradiation at room temperature with $2.0 \mathrm{MeV}$ protons, with fluences in the range from $2 \times 10^{12}$ to $1 \times 10^{14} \mathrm{~cm}^{-2}$, using a Van de Graaff accelerator.

Photoluminescence measurements (PL) were performed for all samples at temperatures ranging from 5 to $300 \mathrm{~K}$, using a FTIR Bruker IFS 66v spectrometer. The $457.9 \mathrm{~nm}$ line of an $\mathrm{Ar}^{+}$laser was used as an excitation source, and the emission from the samples was detected with a Ge detector cooled to liquid nitrogen temperature. The X-ray diffraction and reflection measurements were performed in a high-resolution X-ray diffractometer (Hotbird) [9]. The Hotbird is powered by an $18 \mathrm{~kW}$ rotating anode X-ray generator with a fine focus and has a seven-axes goniometer and is equipped with a linear position-sensitive detector. A parabolic mirror was used in order to enhance the beam quality.

\section{Results and discussion}

The PL spectrum of the as-grown sample A is dominated by two structured bands in the range from 0.95 to $1.08 \mathrm{eV}$ (Fig. 1). Each band consisted of at least four components. The components are separated by the $\mathrm{Si}-\mathrm{Si} \mathrm{TO}$ phonon energy as indicated by the arrows on top of Fig. 1. The two bands are no phonon (NP) and TO phonon assisted transitions related to the radiative recombination of excitons at the GeSi QWs. The appearance of different pairs of NP/TO components is attributed to different $\mathrm{SiGe}$ mixture in the QWs along the multilayer structure. At $0.933 \mathrm{eV}$ a low intensity band is observed, which is attributed to a multiphonon assisted transition. A very weak free and bound excitons related luminescence from the Si substrate and the capping layer is observed at higher energies in the spectrum. At $0.767 \mathrm{eV}$ the "P" optical center appears. This center is related to the presence of $\mathrm{O}$ and is well known from irradiation studies of bulk Si [10]. After passivation, this center almost disappears and the two bands in the range from 0.95 to $1.08 \mathrm{eV}$ show a small increase of the intensity.

For sample B, we observe (Fig. 1) an intense luminescence related to free and bound excitons from the Si substrate and capping layer on the high energy side of the spectrum [10].

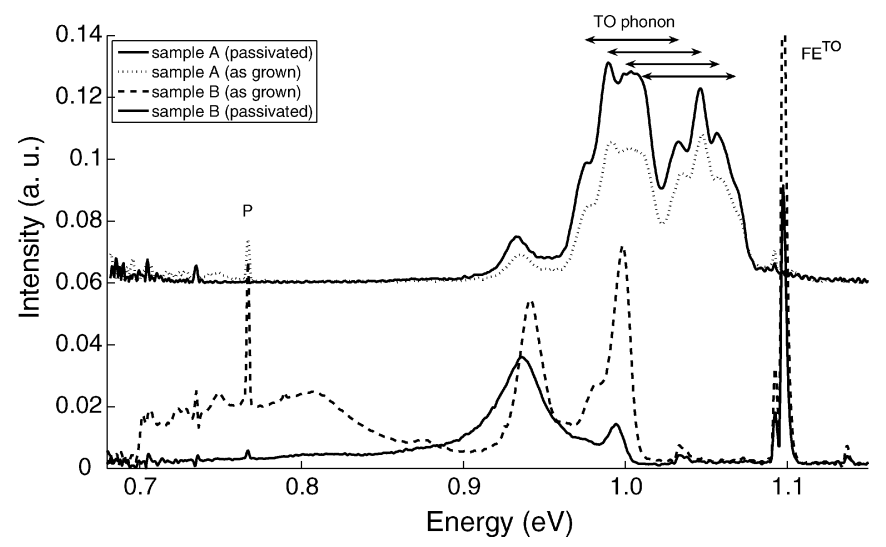

Fig. 1. PL spectra at $12 \mathrm{~K}$ from the as-grown and passivated A and B samples.

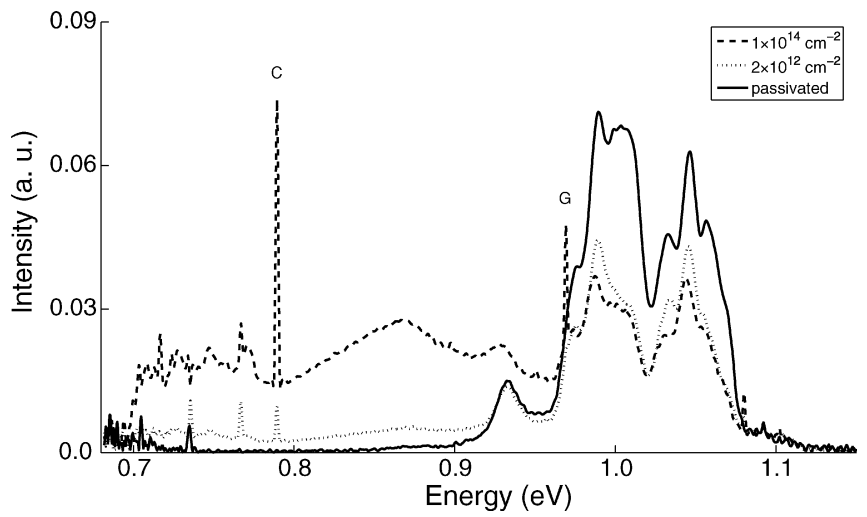

Fig. 2. PL spectra at $12 \mathrm{~K}$ from sample $\mathrm{A}$, as-grown and irradiated to fluences of $2 \times 10^{12}$ and $1 \times 10^{14} \mathrm{~cm}^{-2}$.

At lower energies, four bands centered at 0.998, 0.982, 0.941 and $0.874 \mathrm{eV}$ and corresponding to the zero phonon, TA, TO and multiphonon assisted transitions appear owing to radiative recombination of excitons in a GeSi QW, as was established previously $[11,12]$. These bands are superimposed to a very broad band observed at $h v<1.0 \mathrm{eV}$. The origin for this broad emission may be ascribed to defects because after the passivation with atomic hydrogen it disappears completely. In the PL spectrum of the as-grown sample the "P" center is also observed at lower energies . After passivation, this center is removed from the spectrum which is then dominated by the emission from the GeSi QW.

The RH of the as-grown structures was investigated applying proton irradiation. In Fig. 2 we show the PL spectra obtained for sample A after the two extreme fluences $\left(2 \times 10^{12}\right.$ and $1 \times 10^{14}$ $\mathrm{cm}^{-2}$ ) studied in this work in comparison with the passivated samples. A broad emission appears in almost the whole range of the PL spectrum whose intensity increases with increasing proton fluence. This emission is clearly related to the radiative recombination of charge carriers localized on defects created by the proton irradiation. Two point defects related to the radiation damage are also observed: the "G" center at $0.969 \mathrm{eV}$ and the "C" center at $0.789 \mathrm{eV}$ [10]. The first one involves $\mathrm{C}$ and $\mathrm{Si}$ atoms whereas the second one involves $\mathrm{C}$ and $\mathrm{O}$.

In sample $\mathrm{A}$, we observe that simultaneously with the increase of the defect related emission there is a diminution of the emis-

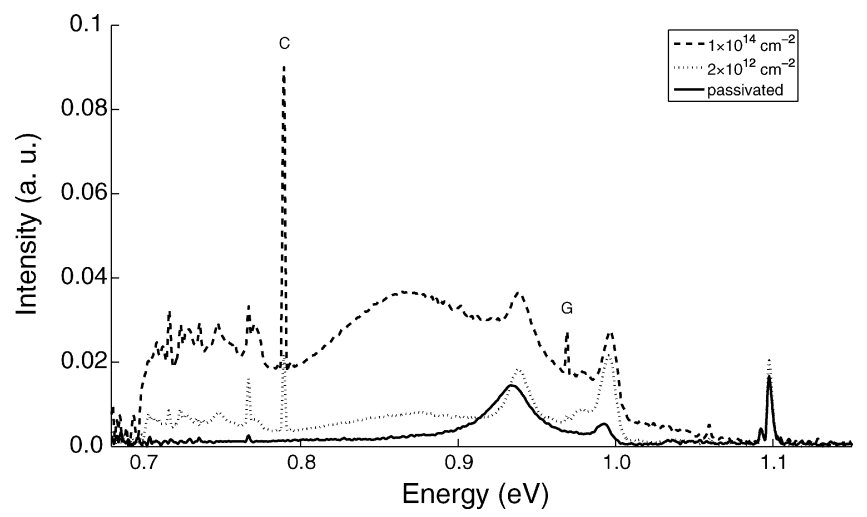

Fig. 3. PL spectra at $12 \mathrm{~K}$ from sample $\mathrm{B}$, as-grown and irradiated with fluences of $2 \times 10^{12}$ and $1 \times 10^{14} \mathrm{~cm}^{-2}$. 

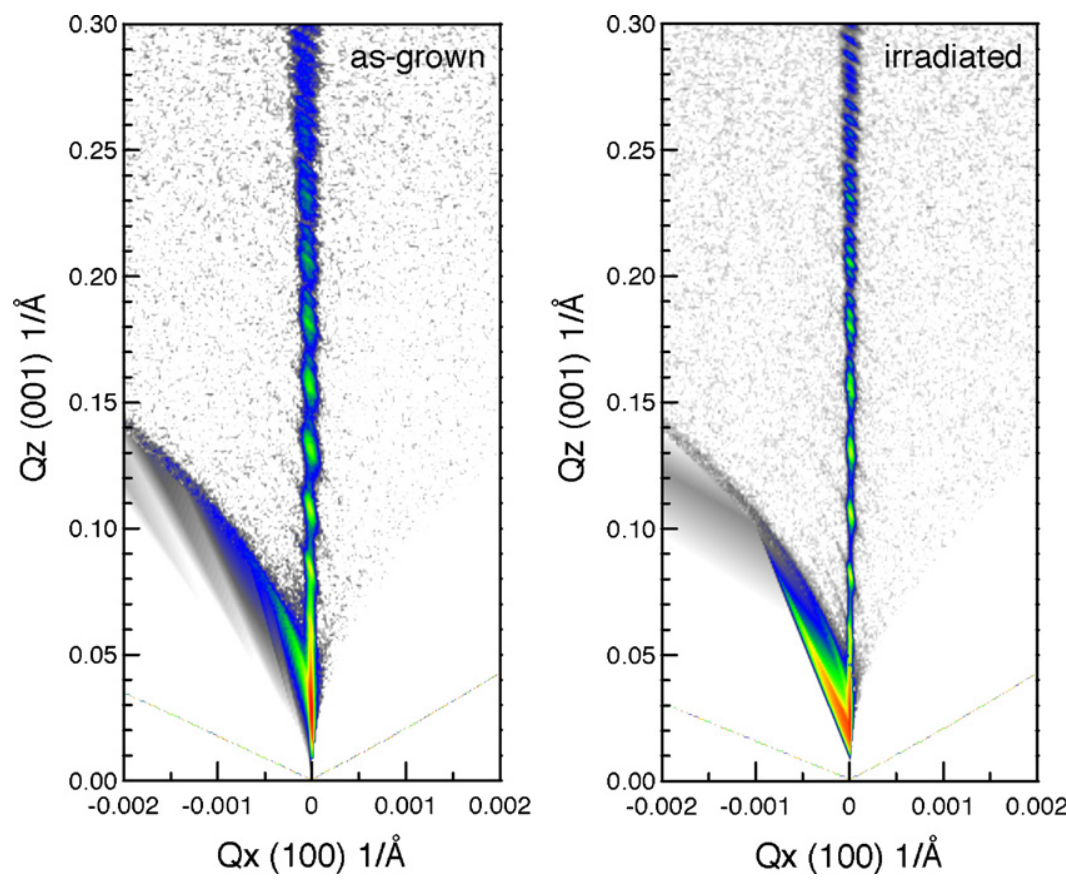

Fig. 4. (Color online) X-ray reflection RSM of as-grown sample A and irradiated with $2.0 \mathrm{MeV}$ protons in the fluence of $1 \times 10^{14} \mathrm{~cm}^{-2}$.

sion intensity from the GeSi QWs. However, even for the highest studied fluence, the emission from the GeSi QWs is clearly present in the PL spectra. This result shows that the GeSi QWs are able to compete with the defects present in the sample for the capture of charge carriers created by the optical pumping. So, an unprecedentedly high radiation resistance was found when compared to the previously studied structures [7].

In sample B, we also observe the appearance of the defect related PL bands. Nevertheless, the PL intensity of the QW continues almost unaffected by irradiation. As discussed above, it is expected that the increase of the density of defects in the QWs will create a high number of non-radiative channels for the charge carriers which will degrade the luminescence from excitons localized in the QWs. However, in sample B the RH of the $\mathrm{PL}$ is even higher than that in sample A. The reason for that is not yet understood.

Fig. 3.

$\mathrm{X}$-ray measurements were done on sample A prior to and after the irradiation to the highest fluence $\left(1 \times 10^{14} \mathrm{~cm}^{-2}\right)$, in order to evaluate the radiation damage in the multilayer structure. The X-ray diffraction reciprocal space map (RSM) of the (0 04) reflection (not shown) performed on the as-grown sample revealed a peak due to the diffraction from the bulk Si and at least four peaks due to the diffraction from the multilayer structure. For the irradiated sample $\left(1 \times 10^{14} \mathrm{~cm}^{-2}\right)$, no significant changes were observed of the positions of the multilayer peaks and of the width at half height. These measurements suggest the absence of a major damage in the multilayer structure due to irradiation.

The X-ray reflection RSMs measured for both the as-grown and irradiated A samples are shown in Fig. 4. For the as-grown sample, we can observe at least six fringes due to the interference from the reflection in the different periods of the multilayer structure. After the irradiation, the fringes were observed at the same positions, confirming a low damage. However, because the X-ray reflection is more sensitive to the interface quality when compared to the X-ray diffraction, it is possible to observe in Fig. 4 a weaker diffuse scattering around the most intense fringes in the irradiated samples. This is interpreted as a less abrupt change in the refractive index between neighboring layers. A possible explanation may be the occurrence of atomic diffusion at the interfaces as a consequence of the generation of defects (interstitials and vacancies) in the different layers and, especially, around the interfaces. In this way, a blurring of the interlayers, without change of the period of the structure, occurs.

The X-ray measurements (diffraction and reflection) are in accordance with the PL measurements in which we still observe the radiative recombination of localized excitons in the $\mathrm{GeSi}$ QWs after the proton irradiation to the highest studied fluence. In spite of a great increase of the density of defects in the sample due to the irradiation treatment, both types of measurements showed that, first, the multilayer structure was not destroyed and, second, that the GeSi QWs were competitive enough in the localization of charge carriers with the defects present in the sample.

\section{Conclusions}

The influence of defects on the optical properties of a single GeSi quantum well deposited on a Si substrate and a diode structure containing a GeSi/Si multilayer structure was investigated. The as-grown samples were passivated with atomic hydrogen and irradiated with $2.0 \mathrm{MeV}$ protons to fluences in the range from $2 \times 10^{12}$ to $1 \times 10^{14} \mathrm{~cm}^{-2}$. For the passivated samples the PL spectra were dominated by the radiative recombination of excitons localized in the GeSi QWs. After the proton irradiation, and in spite of the appearance of a broad band and sharp lines related to defects in Si produced in the irradiation process, 
the emission from the GeSi QWs was observed for all proton fluences. This suggests a low damage of the single $\mathrm{GeSi} Q W$ and the multilayer structure by proton. This interpretation was confirmed by X-ray diffraction and reflection measurements. This behavior shows a high RH of the GeSi QWs under study.

\section{Acknowledgements}

This work was supported by the INTAS project no. 03-515015 and by the RFBR project no. 06-02-08118ofi. J.P. Leitão would like to acknowledge the financial support from Fundação Calouste Gulbenkian and N.P. Stepina a support provided by the SANDiE Network of Excellence for the stay at the University of Aveiro.

\section{References}

[1] F. Schäffler, Semicond. Sci. Technol. 12 (1997) 1515.

[2] R. Leon, G.M. Swift, B. Magness, W.A. Taylor, Y.S. Tang, P. Dowd, Y.H. Zhang, Appl. Phys. Lett. 76 (2000) 2074.
[3] N.A. Sobolev, A. Cavaco, M.C. Carmo, M. Grundmann, F. Heinrichsdorff, D. Bimberg, Phys. Status Solidi (b) 224 (2001) 93.

[4] Ch. Ribbat, R. Sellin, M. Grundmann, D. Bimberg, N.A. Sobolev, M.C. Carmo, Electron. Lett. 37 (2001) 174.

[5] M.B. Huang, J. Zhu, S. Oktyabrsky, Nucl. Instrum. Methods B 211 (2003) 505.

[6] N.A. Sobolev, F.P. Korshunov, R. Sauer, K. Thonke, U. König, H. Presting, J. Cryst. Growth 167 (1996) 502.

[7] N.A. Sobolev, A. Fonseca, J.P. Leitão, M.C. Carmo, H. Presting, H. Kibbel, Phys. Status Solidi (c) (2003) 1267.

[8] A. Fonseca, N.A. Sobolev, J.P. Leitão, E. Alves, M.C. Carmo, N.D. Zakharov, P. Werner, A.A. Tonkikh, G.E. Cirlin, J. Lumin. 121 (2006) 417.

[9] A. Fonseca, N.A. Sobolev, J.P. Leitão, M.C. Carmo, N. Franco, H. Presting, A.D. Sequeira, Mater. Sci. Forum 455/456 (2004) 540

[10] G. Davies, Phys. Rep. 176 (1989) 83.

[11] J.C. Sturm, H. Manoharan, L.C. Lenchyshyn, M.L. Thewalt, N.L. Rowell, J.-P. Nöel, D.C. Houghton, Phys. Rev. Lett. 66 (1991) 1362.

[12] N.L. Rowell, J.-P. Nöel, D.C. Houghton, A. Wang, L.C. Lenchyshyn, M.L. Thewalt, D.C. Perovic, J. Appl. Phys. 74 (1993) 2790. 